\title{
Impacto económico del dengue en Paraguay
}

\section{Dengue economic impact in Paraguay}

\author{
Celia Martínez de Cuellar1, 2, Dolores Lovera', Oscar Merlo' y Antonio Arbo ${ }^{1,2}$
}

'Instituto de Medicina Tropical.

Universidad Nacional de Asunción, Paraguay.

Financiamiento externo: no hubo

Los autores declaran ausencia de conflictos de interés

Recibido (segunda versión): 2 de junio de 2020 / Aceptado: 2 de junio de 2020

\section{Resumen}

Introducción: Desde fines del siglo XX el dengue ha asolado periódicamente al Paraguay. Su elevada morbilidad y mortalidad se traducen en un elevado impacto socio-económico global y una elevada carga en los sistemas de atención médica. Objetivo: Determinar el costo económico y la carga del dengue en el Paraguay. Metodología: Se estimaron los costos directos e indirectos del dengue tanto de casos ambulatorios como hospitalizados. Igualmente fueron calculados los años de vida ajustados por discapacidad (DALY) a partir de la suma de los años de vida perdidos (AVPP) debido a la mortalidad prematura en la población y los años perdidos debido a la discapacidad (DVSP). Resultados: El costo unitario por caso ambulatorio fue de US dólares 52,5 y para pacientes hospitalizados de US dólares 300 con un costo total para los 4 años de US dólares $276.804,864$ y US dólares 23.236,45, respectivamente. Los AVPP fueron 18.469,7 por millón de habitantes; los DVSP de 185.835 y los DALYs 32.676 años por millón de habitantes. Conclusiones: El dengue impone considerables costos tanto para el sector de la salud como para la economía en general en el Paraguay.

Palabras clave: dengue; análisis de costos; Paraguay.

\section{Introducción}

$\mathrm{E}$ 1 dengue es la enfermedad viral transmitida por el mosquito de más rápida propagación en el mundo y que produce un impacto socio-económico y una carga de enfermedad significativa en muchas regiones tropicales y sub-tropicales del planeta ${ }^{1-3}$.

El agente causal virus del dengue (DENV), pertenece al género de los Flavivirus, familia Flaviviridae, y existen cuatro serotipos antigénicamente distintos denominados DENV-1, DENV-2, DENV-3

\begin{abstract}
Background: Since the end of the 20th century, dengue has periodically devastated Paraguay. Its high morbidity and mortality translate into a high global socio-economic impact and a high burden on health care systems. Aim: To determine the economic cost and burden of dengue in Paraguay. Methods: The direct and indirect costs of dengue were estimated in both outpatient and hospitalized cases. Likewise, the years of life adjusted for disability (DALY) were calculated from the sum of the years of life lost (YPLL) due to premature mortality in the population and years lost due to disability (DVSP). Results: The unit cost per ambulatory case was US dollars 52.5 and for hospitalized patients US dollars 300 with a total cost for the 4 years of US dollars 276,804.864 and US dollars 23,236.45, respectively. The AVPP were $18,469.7$ per million inhabitants; the DVSP of 185,835 and the DALYs 32,676 years per million inhabitants. Conclusions: Dengue imposes considerable costs for both the health sector and the economy in general in Paraguay.

Keywords: dengue; costs and cost analysis; Paraguay.
\end{abstract}

y DENV-4. Aunque Aedes aegypti es el principal transmisor de esta arbovirosis, otras especies del género Aedes, como Aedes albopictus, pueden ser vectores de la enfermedad ${ }^{4}$.

Se estima que unos 3.600 millones de personas (cerca de $50 \%$ de la población mundial) viven en zonas en riesgo de la enfermedad, y datos de la Organización Mundial de la Salud revelan que en las últimas décadas su incidencia ha aumentado 30 veces $^{1,5}$. Anualmente, se calcula que ocurren unos 34 millones de casos de dengue clínico, 2 millones de casos de dengue hemorrágico y más de 20.000 muertes $^{3}$. 
de la sumatoria de insumos, medicamentos, laboratorio, métodos auxiliares del diagnóstico, recursos humanos y servicios (alimentación, limpieza) divididos por el número de pacientes hospitalizados en el período. Para ello se revisaron las historias clínicas de pacientes hospitalizados en el Instituto de Medicina Tropical durante los años 2012 y 2013, de donde se obtuvo la cantidad de los insumos descartables, medicamentos, sueros de hidratación parenteral y los servicios de laboratorio, imágenes y ecografías utilizados durante la atención médica hospitalaria o ambulatoria de estos pacientes ${ }^{15}$. Los costos de los medicamentos, insumos y servicios fueron obtenidos de los precios de referencia del MSPBS. Por otro lado, se registró y sumó la remuneración del todo el recurso humano que trabaja en el Instituto de Medicina Tropical en el área ambulatoria y hospitalaria donde se atienden a pacientes con dengue, incluyendo médicos, personal de enfermería, personal de servicios generales y administrativos, la que fue dividida por el número de personas incluidas para obtener el promedio de remuneraciones anuales, valor que luego se dividió por el número de horas trabajadas anualmente, para posteriormente dividir por 60 minutos, obteniendo así una tarifa de remuneraciones por minuto y por estamento. Los costos se analizaron en moneda local (guaraníes), valores que fueron convertidos a USD considerando la paridad cambiaria a mayo de 2013 (1 USD $=4.740$ guaraníes).

Para el cálculo de los costos directos no médicos (por ej. costo de presencia de número de acompañantes, costo de comidas fuera de casa, modo de transporte y alojamiento y sus costos) y de los costos indirectos por caso, se utilizó la literatura internacional, tanto para casos hospitalizados, como para casos ambulatorios ${ }^{13}$.

Los costos de las actividades de prevención y vigilancia dependen básicamente de los fondos del Estado y menos de los gobiernos locales (municipios). Estos costos están integrados por varios componentes, como por ej. las asignaciones realizadas por el gobierno central para las actividades de control del vector. Para ello, se analizaron los costos de los contratos del MSPBS para la compra de materiales (ej. insecticidas, fumigadores). Igualmente, formaron parte del cálculo de los costos los recursos asignados por el gobierno para la vigilancia epidemiológica (tasas pagadas al personal y los gastos en apoyo técnico, por ej. transporte, gastos de viaje y alimentos en el caso de tareas de campo). Un componente substancial de los costos fue el invertido en la contratación de recursos humanos (médicos, enfermeras, personal de apoyo) necesarios para satisfacer las demandas aumentadas causadas por el dengue, así como las inversiones en el fortalecimiento de la red de laboratorios (compra de reactivos para RT-PCR y del antígeno NS1 del dengue, para determinación de IgG e IgM anti-dengue). Estos costos se calcularon en el año 2013. 
Se calcularon los años de vida ajustados por discapacidad para el dengue (DALYs), una medida no monetaria para medir el impacto económico del dengue, cálculo que se realizó a partir de la suma de los años de vida perdidos (AVPP) debido a la mortalidad prematura en la población y los años perdidos debido a la discapacidad (AVPD) para las personas que viven con la enfermedad o sus consecuencias ${ }^{16}$. Los AVPP se obtuvieron a partir del número de muertes multiplicado por la esperanza de vida estándar a la edad en que ocurre la muerte. La fórmula básica para obtener los AVPP fue la siguiente ${ }^{16}$ :

$\mathrm{AVPP}=\mathrm{N} \times \mathrm{L}$

donde:

$\mathrm{N}=$ número de muertes.

$\mathrm{L}=$ esperanza de vida estándar a la edad de la muerte en años.

Para estimar los años de vida saludables perdidos (AVSP) por una causa determinada en un período de tiempo particular, el número de casos incidentes en ese período se multiplica por la duración promedio de la enfermedad y un factor de peso que refleja la gravedad de la enfermedad en una escala de 0 (salud perfecta) a 1 (muerto). La fórmula para obtener los AVSP fue la siguiente ${ }^{16}$ :

$\mathrm{AVSP}=\mathrm{I} \times \mathrm{DW} \times \mathrm{L}$

donde:

$\mathrm{I}=$ número de casos incidentes.

$\mathrm{DW}=$ peso de la discapacidad.

$\mathrm{L}=$ duración promedio del caso hasta la remisión o muerte (años).

El peso por discapacidad (sigla en inglés DW) y duración promedio del caso hasta la remisión o muerte (L) para casos ambulatorios y hospitalizados fueron obtenidos de datos de la literatura médica ${ }^{16}$. Así se calculó el DW para casos ambulatorios 0,051 y la $\mathrm{L}$ de 6 días; para los casos hospitalizados el DW fue de 0,133 y la L 14 días.

\begin{tabular}{|ccccc|}
\hline \multicolumn{4}{|c}{ Tabla 1. Número de casos de Dengue reportados y no reportados. Paraguay 2010-2013 } \\
\hline Año & $\begin{array}{c}\text { Ambulatorios } \\
\text { Casos }\end{array}$ & $\begin{array}{c}\text { Casos reportados } \\
\text { y no reportados }\end{array}$ & $\begin{array}{c}\text { Casos } \\
\text { reportados }\end{array}$ & $\begin{array}{c}\text { Casos reportados } \\
\text { y no reportados }\end{array}$ \\
2010 & 20.288 & 182.592 & 1.075 & 1.505 \\
2011 & 46.069 & 414.621 & 4.900 & 6.860 \\
2012 & 28.676 & 258.084 & 6.061 & 8.485 \\
2013 & 145.249 & 1.307 .241 & 20.679 & 28.951 \\
\hline Total & 240.282 & 2.162 .538 & 32.715 & 45.801 \\
\hline
\end{tabular}

\section{Aspectos éticos}

Este estudio fue aprobado por el Comité de Ética del Instituto de Medicina Tropical. No se obtuvo consentimiento informado de los padres o tutores legales, debido a que se trata de un estudio retrospectivo basado en datos secundarios y no utilizó datos de identificación del paciente.

\section{Resultados}

Durante el período 2010-2013, se reportaron al MSPBS del Paraguay 272.997 casos probables y confirmados de dengue. El número de casos con sub-registro por aplicación del índice de corrección para casos ambulatorios, sumado a los casos registrados, muestra que el número de casos estimados ambulatorios en el período de estudio fue de 2.162 .538 y el número de hospitalizados de 45.801 casos (Tabla 1).

En el período de estudio se observó un incremento substancial de casos entre los años 2010 y 2013, tanto de casos registrados como estimados $(\mathrm{p}<0,001)$ (Tabla 1).

El costo total de la atención incurridos por el sistema de salud para el período 2010-2013 se muestra en la Tabla 2 , exhibiendo los costos médicos directos, costos médicos no directos y costos indirectos por casos ambulatorios y hospitalizados por año, por casos estimados. Esos costos deben aumentarse $25 \%$ para extrapolarse a la situación actual.

En el grupo de pacientes ambulatorios, los costos indirectos fueron mayores que los costos directos en todos los años del estudio, siendo mayores en $18 \%$ en los diferentes años. Así, los costos indirectos por atención ambulatoria en el período de estudio, alcanzaron un costo estimativo de US\$134.077.356 frente a US\$113.533.245 que representó los costos directos (Tabla 2). Al analizar anualmente los costos para la atención ambulatoria de los pacientes y calcularlos en base al presupuesto público total en salud, que en el país es de alrededor de US\$ 800 millones anuales, se pudo constatar que los costos en el año 2010 representaron $2,9 \%$ del presupuesto anual de salud, en el año 2011 fueron $6,6 \%$, en 2012 4,12\% y en 2013 20,9\% del presupuesto anual público $(\mathrm{p}<0,01)$.

Para la atención de casos hospitalizados durante los años 2010-2013, los costos directos representaron la mayor parte de los costos totales. Así, los costos directos médicos totales por atención de casos hospitalizados en todo el período analizado alcanzaron la suma estimativa de US\$13.740.300 lo que fue 45\% mayor que los costos indirectos de los casos hospitalizados (US\$7.648.767), con escasa variación anual (Tabla 2).

Durante los años 2010 a 2013, se observó una letalidad de $0,2 \%$ (520/272.997), de las cuales 52,8\% (275/520) fueron en el sexo masculino. Al analizar los óbitos con- 
Tabla 2. Promedio estimado de costos médicos directos, costos directos no médicos y costos indirectos en dólares. Paraguay $2010-2013$

\begin{tabular}{|c|c|c|c|c|c|c|c|c|}
\hline \multirow[b]{2}{*}{ Año } & \multicolumn{4}{|c|}{ Costos por casos ambulatorios } & \multicolumn{4}{|c|}{ Costos por casos Hospitalizados } \\
\hline & $\begin{array}{l}\text { Médicos } \\
\text { directos }\end{array}$ & $\begin{array}{l}\text { Médicos no } \\
\text { directos }\end{array}$ & Indirectos & Total & $\begin{array}{l}\text { Médicos } \\
\text { directos }\end{array}$ & $\begin{array}{l}\text { Médicos no } \\
\text { directos }\end{array}$ & Indirectos & Total \\
\hline 2010 & 9.586 .080 & 2.008 .512 & 11.320 .704 & 23.371 .776 & 451.500 & 191.135 & 251.335 & 770.560 \\
\hline 2011 & 21.767 .603 & 4.560 .831 & 25.706 .502 & 53.071 .488 & 2.058 .000 & 871.220 & 1.145 .620 & 3.512 .320 \\
\hline 2012 & 13.549 .410 & 2.838 .924 & 16.001 .208 & 33.034 .752 & 2.545 .500 & 1.077 .595 & 1.416 .995 & 4.344 .320 \\
\hline 2013 & 68.630 .153 & 14.379 .651 & 81.048 .942 & 167.326 .848 & 8.685 .300 & 3.676 .777 & 4.834 .817 & 14.822 .912 \\
\hline Total & 113.533 .245 & 23.787 .918 & 134.077 .356 & 276.804 .864 & 13.740 .300 & 5.816 .727 & 7.648 .767 & 23.450 .112 \\
\hline
\end{tabular}

forme al grupo etario, se observó que en los pacientes con edad de $\geq 60$ años y los pacientes con edad de 50 a 59 años, el óbito fue significativamente superior, siendo el riesgo en los $\geq 60$ años de 8,7 veces más. El riesgo atribuible poblacional (RAP) fue de $8,5 \% \mathrm{y}$ el porcentaje del riesgo atribuible poblacional (\% RAP) de 88,9\%. Por otro lado, el número de óbitos en $<1$ año no fue significativo (Tabla 3 ).

$\mathrm{Al}$ analizar los DALYs, la pérdida fue de 1.075 años por millón de habitantes en el 2010, año en que el número de casos de dengue probables y/o confirmados fue de 20.998; esta cifra se incrementó a 4.900 por millón de habitantes en el año 2011 y a 6.093 por millón de habitantes en el 2012. La mayor pérdida se observó en el año 2013, en que el número de casos notificados fue de 145.249 y la pérdida de DALYs estimada fue de 20.608 por millón de habitantes. En el período 2010-2013, los AVPP fueron $18.469,7$ por millón de habitantes y los DVSP fueron 185.835 por millón de habitantes, estimándose una pérdida de DALYs de 32.676 años de discapacidad por dengue por millón de habitantes (Tabla 4).

Los costos totales de las actividades de prevención y supervisión se tuvieron en el año 2013 y se muestran en la Tabla 5. La inversión en la contratación de nuevos recursos humanos para atender la demanda de la epidemia de dengue representó la principal causa de esta fracción de los costos, alcanzando la suma de US\$ 4.191.627, seguida por la inversión en la compra de los insumos (US\$ 1.114.076). Otros componentes fueron menos importantes (Tabla 5).

\section{Discusión}

Durante las últimas décadas, el dengue ha emergido como un grave problema de salud pública en países tropicales y sub-tropicales, incluyendo el Paraguay ${ }^{12,17}$.

Nuestro estudio es el primero en llevarse a cabo para evaluar el costo de la enfermedad del dengue en el país, teniendo en cuenta los casos ambulatorios y hospitalizados entre los años 2010 y 2013. El análisis efectuado, combinando la información disponible sobre casos reportados,

Tabla 3. Óbitos según grupo de edad.

\begin{tabular}{lcc}
\hline Grupo de Edad & $\mathbf{n}$ & $\%$ \\
$<1$ año & 10 & $1,9^{*}$ \\
\hline a 4 & 5 & 1,0 \\
\hline 5 a 14 & 20 & 3,8 \\
\hline 15 a 19 & 18 & 3,5 \\
\hline 20 a 39 & 101 & 19,4 \\
\hline 40 a 49 & 68 & 13,1 \\
\hline 50 a 59 & 79 & $15,2^{* *}$ \\
\hline$>60$ & 219 & $42,1^{* * *}$ \\
\hline Total & 520 & 100,0 \\
\hline${ }^{*} p=0,5 .{ }^{* *} p<0,00001 .{ }^{* * *} p<0,00001$. & \\
\hline
\end{tabular}

Tabla 4. Años de Vida Potencialmente Perdidos (AVPP) y Días de Vida Saludable Potencialmente Perdidos (DVSP), según año de ocurrencia

\begin{tabular}{ccccc}
\hline Año & Casos notificados & AVPP* & DVSP* & DALYs \\
2010 & 20.288 & 653,9 & $15.117,2$ & 1.075 \\
2011 & 46.069 & $3.556,1$ & $32.320,6$ & 4.900 \\
2012 & 28.676 & $3.485,8$ & $36.041,2$ & 6.093 \\
2013 & 145.249 & $10.773,9$ & $102.356,8$ & 20.608 \\
\hline Total & 272.997 & $18.469,7$ & $185.835,8$ & 32.676 \\
\hline *por millón de habitantes. & & & \\
\hline
\end{tabular}

Tabla 5. Costos en inversiones durante la epidemia de dengue año 2013

Tipo de inversión

Contratación de nuevos recursos humanos para la epidemia

Compra de insumos para control de vectores

Inversión en vigilancia epidemiológica

Fortalecimiento de la red de laboratorios

Total
Costo invertido en US\$

4.191 .627

1.114 .076

48.464

168.795

5.522 .962 
niveles de sub-registro y costo por caso, muestra que la carga económica anual del dengue en el Paraguay es sustancial, pero con variaciones anuales dependiendo de la intensidad de la epidemia.

Estudios recientes estimaron la carga económica del dengue en ciertos países del Sudeste de Asia. Los costos han variado con la envergadura de las epidemias en los diferentes países. Así, en Camboya, en un año con carga habitual de dengue, los costos calculados fueron de US\$3.327.284; en cambio, en un año epidémico, esa cifra casi se quintuplica (US\$14.429.513) ${ }^{18}$. Esta figura es mucho mayor para otros países del sudeste asiático como Malasia y Tailandia, en los que se estimó que el dengue impone un costo anual de US\$ 42,4 y US\$ 53,1 millones respectivamente, representando en estos países 41,3 y 49\% del costo total del en salud (19-21). Otros estudios estimaron un costo de US\$ 8 millones en Camboya, entre 2006 y 2008; y de US\$ 103,4 millones por año en Malasia ${ }^{17}$.

Suaya JS y cols..$^{20}$, analizaron el costo del dengue en países de las Américas como Brasil, El Salvador, Guatemala, Panamá y Venezuela. Este grupo estimó que el costo total promedio de un caso ambulatorio no fatal fue de US\$ 514, en tanto que el costo promedio de un caso hospitalizado no fatal fue de US\$ $1.491^{20}$; en promedio, un caso hospitalizado costó tres veces más que un caso ambulatorio. Sumando los pacientes ambulatorios y hospitalizados, y contabilizando el riesgo de muerte, el costo total de un caso de dengue fue de US\$ 828. Al sumar esta cifra con el costo promedio anual de los casos de dengue oficialmente reportados en dichos países, estudiados en el período 2001-2005 (532.000 casos), se obtuvo un costo de US\$ 440 millones para el dengue oficialmente notificado. Este estimativo es muy conservador e ignora, no solamente la falta de notificación de todos los casos, sino también, los costos sustanciales asociados con los programas de vigilancia y control de los vectores del dengue. Este estudio demostró que un episodio de dengue tratado impone costos sustanciales, tanto en el sector de la salud como en la economía global ${ }^{20}$.

Nuestros resultados son consistentes con las observaciones de otros países con actividad periódica de dengue; revela que las epidemias de dengue durante los años 2010 a 2013 demandaron un costo de US\$276.804.864 por casos ambulatorios y US\$23.450.112 por casos hospita- lizados. Además, la carga económica para el control de vectores, así como para la contratación de nuevos recursos humanos para hacer sostenible la alta demanda en salud de una epidemia, hacen que los costos del dengue sean significantes. Países como Brasil, igualmente reportan este alto $\operatorname{costo}^{22}$. Desde que el presupuesto público total en salud en el país es alrededor de US $\$ 800$ millones, los costos económicos del dengue son significantes. Por lo tanto, si los costos del dengue pudieran reducirse, parte de los ahorros podrían ser re-ubicados a otros servicios de salud.

Las consecuencias del dengue, medidas en términos de DALYs, son sustanciales, siendo nuestras estimaciones del número anual de DALY relacionados con el dengue coherentes con reportes de otros países. Así, Shepard DS y cols. ${ }^{13}$, estimaron para Brasil que los DALYs causados por el dengue entre los años 2000 y 2007 fueron de 26.492 años por millón de habitantes y para América 72.772. En nuestro estudio, los AVPP fueron 18.469,7, los DVSP fueron 185.835, y los DALYs 32.676 años de discapacidad por dengue por millón de habitantes, lo que refleja el tremendo impacto económico de la enfermedad en el Paraguay.

El presente estudio posee varias limitaciones. En este sentido, los factores de corrección para estimar el subregistro de casos son estimaciones basadas en conjuntos limitados de datos de otros países. Igualmente, no se consideró otros impactos del dengue, por ejemplo, en el turismo, un negocio incipiente en el Paraguay, y en la disminución de la actividad comercial durante las epidemias, entre otros.

Finalmente, nuestro análisis presenta las estimaciones de la carga de dengue en el Paraguay y proporciona datos económicos que no estaban disponibles anteriormente. Nuestros resultados son conservadores, porque algunos componentes importantes no se incluyeron en los costos relacionados con el dengue. En ausencia de vacunación, y siendo los programas de control del vector la estrategia básica para mitigar la propagación del dengue, esta enfermedad seguirá produciendo una carga económica y social considerable en el Paraguay, lo que se refleja en el costo total de la enfermedad. Se requiere de acciones multisectoriales sostenibles para reducir el impacto económico y social del dengue en la población paraguaya.

\section{Referencias bibliográficas}

1.- Wider-Smith A, Ooi E E, Horstick O, Wills B. Dengue. Lancet 2019; 393 (10169): 350-63. doi: 10.1016/S0140-6736(18)32560-1.

2.- Guzmán M G, Kouri G. Dengue: an update Lancet Infect Dis 2002; 2 (1): 33-42. doi: 10.1016/s1473-3099(01)00171-2.

3.- Castro M C, Wilson M E, Bloom D E. Disease and economical burdens of dengue. Lancet Infect Dis 2017; 17 (3): e70-e78. doi: 10.1016/ S1473-3099(16)30545-X.

4.- Chang G-J. Molecular biology of dengue viruses. In: Gubler DJ, Kuno G, eds. Dengue and dengue hemorrhagic fever. London: $\mathrm{CAB}$ International; 1997. p. 175-98

5.- Murray N E, Quam M B, Wilder-Smith A. Epidemiology of dengue: past, present and future prospects. Clin Epidemiol 2013; 5 (1): 299-309. doi: 10.2147/CLEP.S34440.

6.- Ministerio de Salud Pública y Bienestar 
Social Dirección General de Vigilancia de la Salud. Enfermedades transmitidas por vectores. Boletín Epidemiológico. 2016; 30 11. Disponible en: http://vigisalud.gov.py/ boletines /21_09_2016_11_18_01_BoletinEpidemiologico_SE-34.pdf.

7.- Zubieta-Zavala A, López-Cervantes M, Salinas-Escudero G, Ramírez-Chávez A, Castañeda J R, Hernández-Gaytán S I, et al. Economic impact of dengue in Mexico considering reported cases for 2012 to 2016. PLoS Negl Trop Dis 2018; 12 (12):e0006938. https://doi.org/10.1371/journal.pntd.0006938.

8.- Brathwaite Dick O, San MartÍn J L, Montoya R, Del Diego J, Zambrano B, Dayan G H. The history of Dengue outbreaks in the Americas. Am J Trop Med Hyg 2012; 87 (4): 584-93. doi: 10.4269/ajtmh.2012.11-0770.

9.- San Martín J L, Brathwaite O, Zambrano B, Solórzano J O, Bouckenooghe A, Dayan $\mathrm{G} \mathrm{H}$, et al. The epidemiology of dengue in the Americas over the last three decades: a worrisome reality. Am J Trop Med Hyg 2010; 82 (1): 128-35. PMID: 20065008.

10.- Rice D P. Estimating the cost-of-illness. Washington, DC: US Department of Health, Education, and Welfare, Public Health Service; 1966.

11.- Hung T M, Clapham H E, Bettis A A, Cuong H Q, Thwaites G E, Wills B A, et al. The estimates of the health and economic burden of Dengue in Vietnam. Trends Parasitol 2018; 34 (10): 904-18. doi: 10.1016/j.pt.2018.07.007.

12.- WHO. Dengue: guidelines for diagnosis, treatment, prevention and control. Geneva, Switzerland: WHO; 2009.

13.- Shepard D, Coudeville L, Halasa Y A, Zambrano B, Dayan G H. Economic impact of dengue illness in the Americas. Am J Trop Med Hyg 2011; 84 (2): 200-7. doi: 10.4269/ ajtmh.2011.10-0503.

14.- Dirección Nacional de Estadisticas y Censos. Atlas Demográfico del Paraguay. 2012. Disponible: https://www.dgeec.gov.py/ Publicaciones/Biblioteca/atlas-demografico/ Atlas\%20Demografico\%20del\%20 Paraguay,\%202012.pdf.

15.- Peña-Kieninger M, Gimenez-Caballer E, Arbo-Sosa A, Torres-Amarilla C, Jauregui B, Januz C B, et al. Cost-effectiveness analysis of pneumococcal conjugate vaccine introduction in Paraguay. Vaccine 2015; 33 (Supl 1): A143-A153. doi: 10.1016/j. vaccine.2014.12.078.

16.- Murray C J. Disability-adjusted life years (DALYs) for 291 diseases and injuries in 21 regions, 1990-2010: a systematic analysis for the Global Burden of Disease Study 2010. Lancet 2012; 380 (9859): 2197-223. doi: 10.1016/S0140-6736(12)61689-4.
17.- Stanaway J D, Shepard D S, Undurraga E, Halasa Y A, Coffeng L E, Brady O J, et al. The global burden of dengue: an analysis from the Global Burden of Disease Study 2013. Lancet Infect Dis 2016; 16 (6): 712-23. doi: 10.1016/ S1473-3099(16)00026-8.

18.- Beauté J, Vong S. Cost and disease burden of dengue in Cambodia. BMC Public Health 2010; 10: 521. https://doi.org/10.1186/1471-2458-10521.

19.- Okanurak K, Sorrumani S, Indaratna K. The cost of dengue hemorrhagic fever in Thailand. Southeast Asian J Trop Med Public Health. 1997; 28 (4): 711-7. PMID: 9656390.

20.- Suaya J S, Shepard D S, Siqueira J B, Martelli C T, Lum L C S, Tan L H, et al. Cost of dengue cases in eight countries in the Americas and Asia: a prospective study. Am J Trop Med Hyg 2009; 80 (5): 846-55. PMID: 19407136.

21.- Lim L H, Vasan S S, Birgelen L, Murtola T M, Gong H-F, Field R W, et al. Immediate cost of dengue to Malaysia and Thailand: An estimate. Dengue Bulletin 2010; 34: 65-76. https://apps. who.int/iris/handle/10665/170972.

22.- Pepin K M, Marques-Toledo C, Scherer L, Morais M M, Ellis B, Eiras A E. Costeffectiveness of novel system of mosquito surveillance and control, Brazil. Emerg Infect Dis 2013; 19 (4):542-50. doi: 10.3201/ eid1904.120117. 